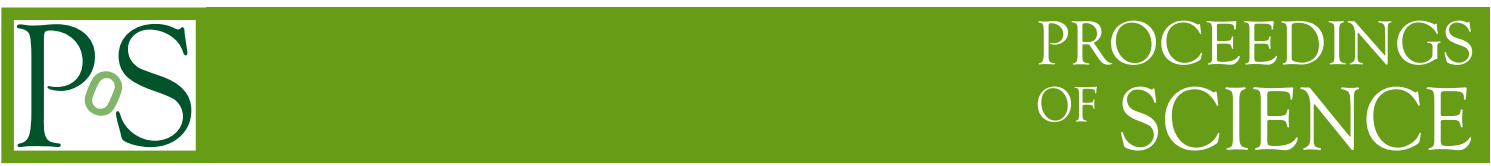

\title{
Results overview from the DAMPE space mission in orbit
}

\section{Kyratzis ${ }^{a, b, *}$ for the DAMPE collaboration}

${ }^{a}$ Gran Sasso Science Institute (GSSI), Via Iacobucci 2, 67100, L’Aquila, Italy

${ }^{b}$ INFN, Laboratori Nazionali del Gran Sasso (LNGS), 67100 Assergi, L'Aquila, Italy

E-mail: dimitrios.kyratzis@gssi.it

The Dark Matter Particle Explorer (DAMPE), is a space-borne detector designed for precise galactic Cosmic Ray (CR) studies in a wide energy range (up to $100 \mathrm{TeV}$ ), along with detailed measurements of high-energy gamma-rays and indirect searches of Dark Matter (DM) annihilation/decay to detectable particles. The satellite was launched into a sun-synchronous orbit at 500 $\mathrm{km}$, on December 17th 2015 and has been successfully acquiring data ever since. The instrument consists of four sub-detectors, namely: a Plastic Scintillator Detector (PSD), a Silicon TracKerconverter (STK), a deep Bismuth Germanate (BGO) calorimeter $32 X_{0}, 1.6 \lambda_{I}$ ) and a NeUtron Detector (NUD).

DAMPE provided valuable insight on the cosmic-ray electron+positron (CRE) spectrum, unveiling a clear spectral break at $0.9 \mathrm{TeV}$ with unprecedented energy resolution. Moreover, recent results regarding $\mathrm{CR}$ protons reveal a spectral hardening (at few hundred $\mathrm{GeV}$ ) followed by a softening feature at $10 \mathrm{TeV}$. These features are well described by a smoothly-broken power law (SBPL), which differs from the paradigm of a unique power law (PL) spectrum extending up to PeV energies, hence necessitating a careful reconsideration of prevailing CR models. Additional insights concerning spectral measurements of helium, medium (i.e, boron, carbon, nitrogen, oxygen) and heavier mass nuclei (iron), will be presented in this work. Preliminary results on secondary-over-primary ratios (i.e., $\mathrm{B} / \mathrm{C}$ and $\mathrm{B} / \mathrm{O}$ ) crucial in deciphering the nature of $\mathrm{CR}$ propagation in the Galaxy, will also be discussed, including a general synopsis of the mission status.

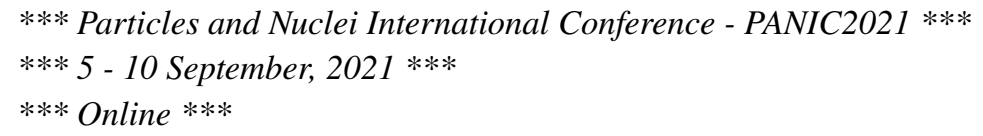

${ }^{*}$ Speaker 


\section{Introduction}

Nowadays, Cosmic Ray (CR) detection remains at the forefront of intense research, represented by a multitude of sophisticated experiments aiming towards the clarification of their origin, acceleration and propagation mechanisms in the Universe [1]. Although crucial information has been well-received from indirect (ground-based) experiments over the years, there is an imminent need to explore highly energetic CR particles and gamma-rays by means of direct observations, carried out by space-borne detectors. In that regard, distinct features can be revealed in the energy spectra along with a consistent picture concerning the evolution of mass composition with energy. Consequently, the Dark Matter Particle Explorer (DAMPE) was introduced as a space-borne instrument [2], to provide insight on galactic CR Physics with precise measurements of electrons/positrons (up to 10 $\mathrm{TeV}$ ), protons and nucleonic spectra ( hundreds of TeV). At the same time, DAMPE offers a great opportunity to enhance our understanding in topics concerning gamma ray astronomy throughout a broad energy range, while distinguishing particles originating from astrophysical sources over possible dark matter annihilation products (due to inherent differences in both spectra). The detector was successfully launched on December 17th, 2015 in a low-Earth orbit (LEO) of $\sim 500 \mathrm{~km}$ and continues its stable operations ever since.

\section{DAMPE: Detector description}

DAMPE (highlighted in Fig. 1a) consists of four sub-detectors (starting from the top): a Plastic Scintillator Detector (PSD) [3], a Silicon TracKer-converter (STK) [4], a deep Bismuth Germanate (BGO) calorimeter (32 $X_{0}$ and $\left.1.6 \lambda_{I}\right)$ [5], along with a NeUtron Detector (NUD) [6]. A highly-energetic particle impinging on DAMPE should initially interact with the PSD; configured to precisely measure the charge as well as providing an effective veto in the case of gamma rays. Subsequently, the particle should traverse the STK, which accurately reconstructs its track and converts incoming gamma-rays into electron-positron pairs (due to the inclusion of tungsten layers), while also providing an additional charge measurement. Afterwards, the propagating particle should deposit its energy inside the BGO calorimeter, by means of electromagnetic or hadronic cascades, therefore yielding valuable information regarding its overall identification. An additional level of electron-hadron discrimination is achieved with the NUD, being instrumental at $\mathrm{TeV}$ energies.

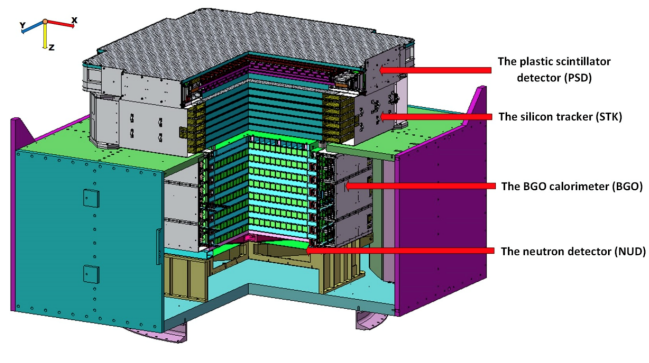

(a)

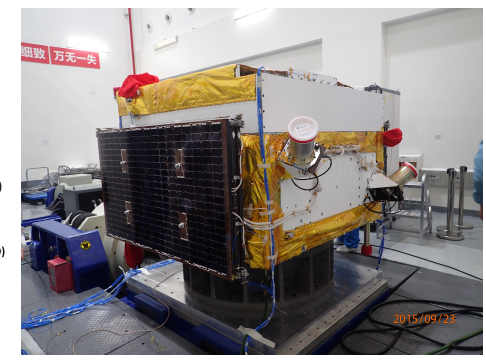

(b)

Figure 1: (a) Schematic illustration of DAMPE along with its four sub-detectors, (b) Full detector configuration subjected to various space-readiness tests before flight. 


\section{Overview of $\mathrm{CR}$ results and ongoing work}

\subsection{Fine structure of the all-electron spectrum}

The all-electron component $\left(\mathrm{e}^{+} \mathrm{e}^{-}\right)$comprises $1 \%$ of total CRs, with a spectrum well described by a single power-law, consistent with recent measurements. Initially, ground-based Imaging Atmospheric Cherenkov Telescopes (IACTs) HESS and VERITAS had shown hints of a spectral break in the $\mathrm{e}^{+}+\mathrm{e}^{-}$spectrum near the TeV range, with VERITAS extending its results up to 20 TeV. In 2017, DAMPE [7] published results regarding the all-electron spectrum in the energy range of $25 \mathrm{GeV}$ to $4.6 \mathrm{TeV}$ with great precision and large acceptance (from 530 days of data) (shown in Fig. 2a), thus leading to the first direct evidence of a spectral break at $\approx 1 \mathrm{TeV}$. The aforementioned electrons experience energetic losses during propagation, which, in addition to the $\mathrm{CR}$ diffusion coefficient value (extracted by $\mathrm{B} / \mathrm{C}$ measurements) leads to an estimation of their production origin, within approximately $100 \mathrm{pc}$ (local neighborhood).

\subsection{Protons and helium nuclei}

Constituting the majority of CRs, protons [8] and helium nuclei [9] were thoroughly investigated by DAMPE. In the case of protons, a data sample of 30 months was analyzed in the energy range of $40 \mathrm{GeV}$ to $100 \mathrm{TeV}$, leading to the detection of a spectral hardening around $500 \mathrm{GeV}$ (consistent with previous observations) along with a novel softening featured approximately at $14 \mathrm{TeV}$ (Fig. 2b). The aforementioned characteristics were precisely described by a smoothly-broken power-law (SBPL) model above the observed hardening, hence ruling-out the previously favored paradigm of a single power-law governing $\mathrm{CR}$ spectra up to $\mathrm{PeV}$ energies. Furthermore, DAMPE presented a detailed spectral measurement of helium nuclei in the energy range of $70 \mathrm{GeV}$ to $80 \mathrm{TeV}$, extracted from 54 months of flight data. In that sense, an analogous picture with the proton case was revealed; a hardening feature around $1 \mathrm{TeV}$ in accordance with previous results, followed by a spectral softening around $34 \mathrm{TeV}$ (seen in Fig. 2c). These novel observations necessitate a careful reconsideration (or update) of prevailing CR models, consequently aiding towards an extended understanding of the intricate details regarding the origin and acceleration mechanism of galactic CRs. Ongoing work includes a joint analysis of proton and helium nuclei $(\mathrm{p}+\mathrm{He})$, with the clear advantage of increased statistics, higher achieved energies, along with lower background contamination, hence allowing for direct comparison with results obtained by ground-based experiments.

\subsection{Advancements on Medium and Heavy mass CR nuclei}

Following recent results on above-stated works, the DAMPE collaboration is heavily involved in various analyses concerning both primary and secondary CR nuclei ranging from lithium all the way up to iron, along with insights on secondary-over-primary ratios. Specifically, ongoing studies concern lithium, beryllium and boron CR nuclei, being secondary spallation/fragmentation products of heavier nuclei such as carbon, nitrogen and oxygen interacting with the Interstellar Medium (ISM) during propagation. Consequently, great emphasis is given to ongoing spectral measurements of primaries such as carbon and oxygen (together with the partially primary nitrogen) throughout an extended energy range (several $\mathrm{TeV} / \mathrm{n}$ ), in order to possibly reveal features analogous to previous analyses. An idea of current results regarding charge contributions obtained from the DAMPE 


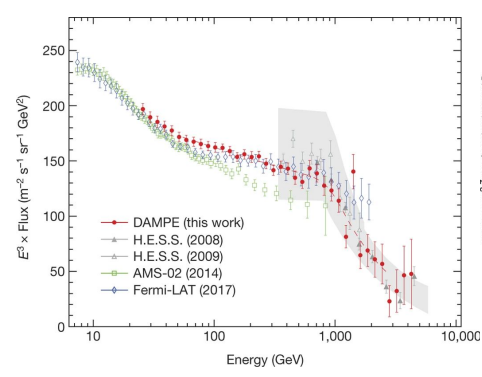

(a)

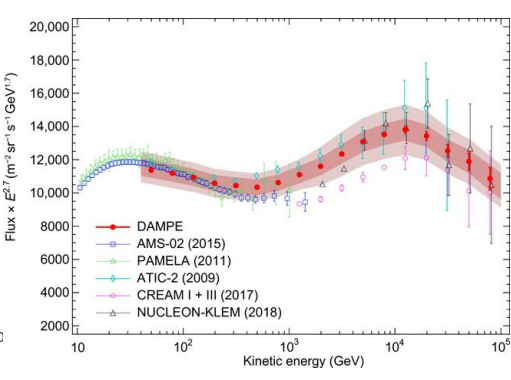

(b)

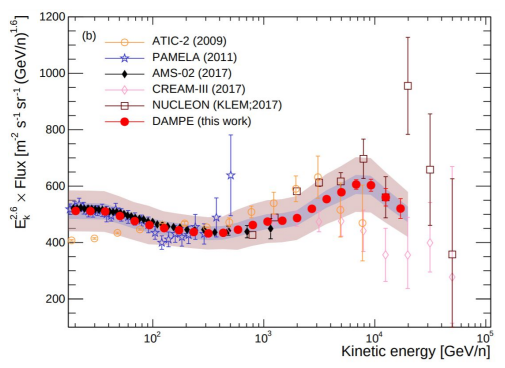

(c)

Figure 2: DAMPE spectral measurements concerning: (a) electrons + positrons, (b) protons, (c) helium nuclei, multiplied by $\mathrm{E}^{3}, \mathrm{E}^{2.7}$ and $\mathrm{E}^{2.6}$ respectively.

PSD (in the BCNO group), can be seen in Fig. 3a. Additionally, bringing together analyses of primary $(\mathrm{C}, \mathrm{O})$ and secondary $(\mathrm{Li}, \mathrm{Be}, \mathrm{B}) \mathrm{CR}$ nuclei in the form of secondary-over-primary ratios, will offer a unique probe of the CR propagation mechanism in the ISM. In that sense, preliminary results from ongoing $\mathrm{B} / \mathrm{C}$ ratio works in a restricted energy range (up to $400 \mathrm{GeV} / \mathrm{n}$ ), are illustrated in Fig. 3b. From the point of medium mass CR nuclei, moving towards the heaviest stable components produced during supernova nucleosynthesis, iron is one of the most abundant primary CR components currently studied. Numerous analysis challenges arise from such massive nuclei impinging on space-borne instruments, due to severe fragmentation effects inside the active detector volume. Said contamination effects are currently being evaluated in order to acquire the purest possible sample.

Due to its increased acceptance and enhanced detector capabilities, DAMPE can expand previous CR measurements in the multi-TeV region (being an "uncharted territory" from the directdetection perspective) with high precision and low background, thus proving to be decisive in deciphering open questions concerning the origin, acceleration and propagation mechanisms of galactic CRs.

\section{Summary}

The Dark Matter Particle Explorer (DAMPE), is a space-borne instrument currently in stable data-acquisition since December 2015, specifically designed for precise galactic Cosmic Ray studies in a wide energy range (up to $100 \mathrm{TeV}$ ), along with detailed measurements of high-energy gamma-rays and indirect searches of Dark Matter (DM) annihilation/decay to detectable particles. DAMPE published insightful results on the $\mathrm{e}^{+}+\mathrm{e}^{-}$(CREs) spectrum, in the energy range from 25 $\mathrm{GeV}$ to $4.6 \mathrm{TeV}$, revealing a spectral break at $\sim 1 \mathrm{TeV}$. Furthermore, DAMPE measured the proton flux from $40 \mathrm{GeV}$ to $100 \mathrm{TeV}$, confirming the detection of a spectral hardening around $500 \mathrm{GeV}$ along with the onset of a softening, featured approximately at $14 \mathrm{TeV}$. In the case of helium nuclei, the measured spectrum extending from $70 \mathrm{GeV}$ to $80 \mathrm{TeV}$, showed a hardening at $\mathrm{TeV}$ energies along with strong evidence of a spectral softening at approximately $34 \mathrm{TeV}$. The aforementioned characteristics were precisely described by smoothly-broken power-law (SBPL) models above the observed hardenings, hence ruling-out the previously favored paradigm of a single power-law. On- 
going DAMPE studies include the analyses of: $\mathrm{p}+\mathrm{He}, \mathrm{Li}, \mathrm{Be}, \mathrm{B}, \mathrm{C}, \mathrm{O}$ and $\mathrm{Fe}$ nuclei along with insights on secondary-over-primary ratios like $\mathrm{B} / \mathrm{C}$ and $\mathrm{B} / \mathrm{O}$.

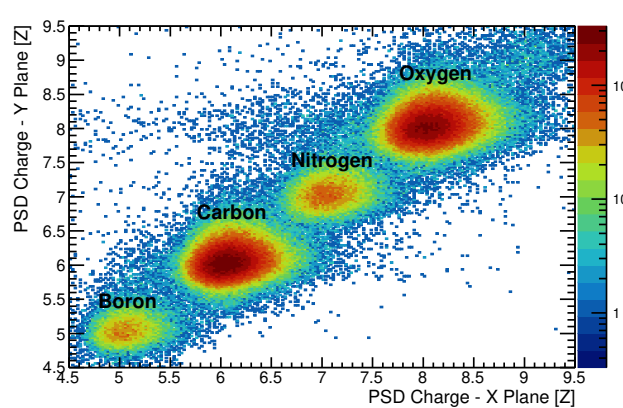

(a)

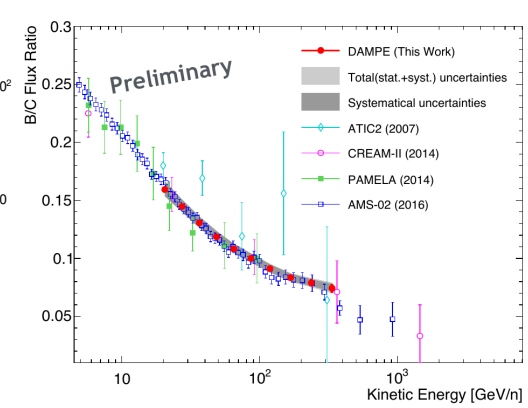

(b)

Figure 3: (a) Charge spectra illustrating flight data of boron, carbon, nitrogen and oxygen in the PSD (Y vs $\mathrm{X}$ plane), (b) Preliminary results on the B/C ratio (up to $\sim 400 \mathrm{GeV} / \mathrm{n}$ ) from 5 years of DAMPE data.

\section{Acknowledgements}

The author would like to thank all members of the DAMPE collaboration for supporting this work with valuable comments and fruitful discussions.

\section{References}

[1] R. Aloisio, P. Blasi, I. De Mitri, S. Petrera, Selected Topics in Cosmic Ray Physics. In: Aloisio R., Coccia E., Vissani F. (eds) Multiple Messengers and Challenges in Astroparticle Physics. Springer, Cham. (2018)

[2] J. Chang et al. [DAMPE Collaboration], The Dark Matter Particle Explorer mission, Astropart. Phys. 95 (2017) 6-24.

[3] T. Dong et al., Charge measurement of cosmic ray nuclei with the plastic scintillator detector of DAMPE, Astropart. Phys. 105 (2019) 31-36.

[4] P. Azzarello et al., The DAMPE silicon-tungsten tracker, Nucl. Instrum. Meth. A 831 (2017) 379

[5] Z-Y. Zhang et al., The calibration and electron energy reconstruction of the BGO ECAL of the DAMPE detector, Nucl. Instrum. Meth. A. 836 (2016) 98

[6] Y-Y. Huang et al., Calibration and performance of the neutron detector onboard of the DAMPE mission, Res. Astron. Astrophys. 20 (2020) 153

[7] G. Ambrosi et al. [DAMPE Collaboration], Direct detection of a break in the teraelectronvolt cosmic-ray spectrum of electrons and positrons, Nature, 552 (2017) 63

[8] Q. An et al. [DAMPE Collaboration], Measurement of the cosmic ray proton spectrum from $40 \mathrm{GeV}$ to $100 \mathrm{TeV}$ with the DAMPE satellite, Sci. Adv., 5 (2019) eaax3793

[9] F. Alemanno et al. [DAMPE Collaboration], Measurement of the Cosmic Ray Helium Energy Spectrum from $70 \mathrm{GeV}$ to $80 \mathrm{TeV}$ with the DAMPE Space Mission, Phys. Rev. Lett, 126 (2021) 201102 